\title{
Ichthyosis Prematurity Syndrome: A Rare Form but Easily Recognizable Ichthyosis
}

\author{
Sultan Al-Khenaizan ${ }^{a} \quad$ Asma AlSwailem $^{b} \quad$ Mohammed Ali AlBalwi ${ }^{c}$ \\ aDivision of Dermatology, Department of Pediatrics, King Abdulaziz Medical City, National \\ Guard Health Affairs, Riyadh, Saudi Arabia; bivision of Dermatology, King Saud Medical \\ City, Riyadh, Saudi Arabia; 'Department of Pathology and Laboratory Medicine, King Saud \\ Bin Abdulaziz University for Health Sciences, King Abdullah International Medical Research \\ Center, King Abdulaziz Medical City, Riyadh, Saudi Arabia
}

\section{Keywords}

Ichthyosis prematurity syndrome · Solute carrier family 27 member $4 \cdot$ Verruciform hyperkeratotic plaques - Cobblestone appearance · Premature birth · Neonatal asphyxia

\section{Abstract}

Ichthyosis prematurity syndrome is a rare autosomal recessive genodermatosis that is associated with mutations in the SLC27A4 gene. Its onset occurs in early childhood and presents with the clinical triad of premature birth, thick caseous desquamating epidermis, and neonatal asphyxia. Here, we describe a prematurely born baby patient (33 weeks of gestation) with a homozygous variant at the initiation codon site (c.1 A> G, p.Met1Val) in the SLC27A4 gene to raise awareness of this rare syndrome despite its distinctive features as we believe it is still underdiagnosed.

\section{Introduction}

Ichthyosis prematurity syndrome (IPS, OMIM608649) is a rare autosomal recessive disorder characterized by the clinical triad of premature birth, ichthyosis, and neonatal asphyxia [1]. It is caused by a mutation in SLC27A4 (solute carrier family 27 member 4, which encodes fatty acid transport protein 4 [FATP4]) [2]. Here, we describe an IPS patient with a homozygous pathogenic variant at the initiation codon site (c.1 A>G, p. Met $1 \mathrm{Val}$ ) in the $S L C 27 A 4$ gene to raise awareness of this rare syndrome despite its distinctive features as we believe it is still underdiagnosed. 


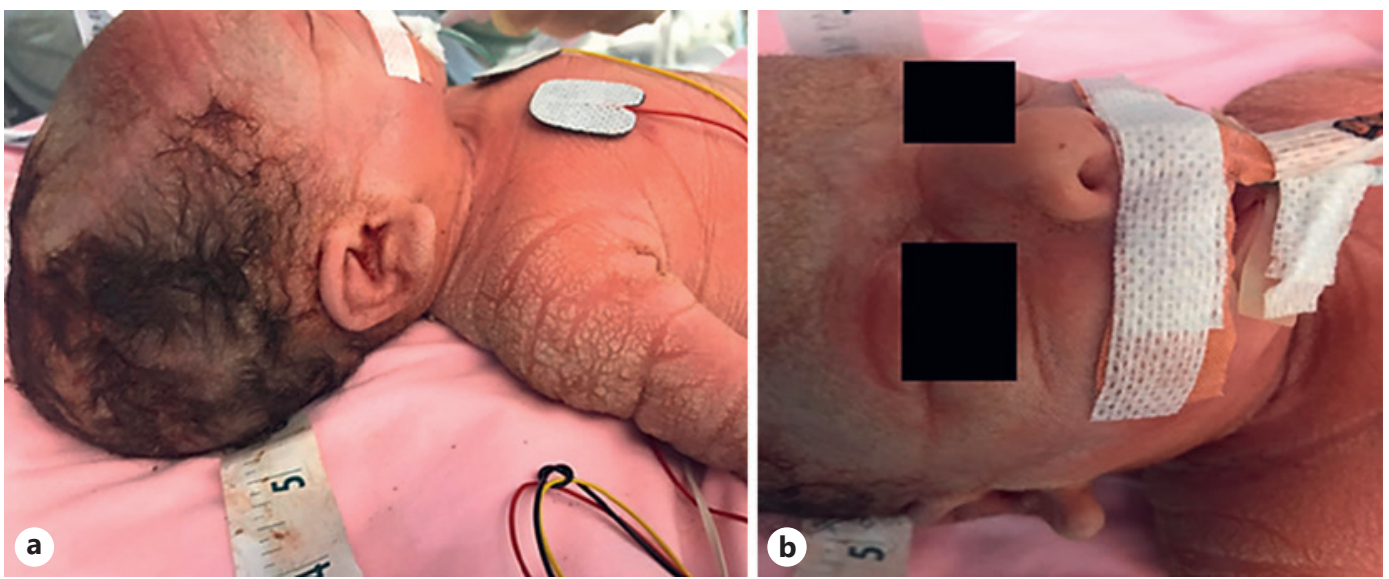

Fig. 1. Photograph of the patient at birth shows a multiple thick verruciform hyperkeratotic plaques with cobblestone appearance covering all of his body including the scalp and $\mathbf{b}$ cobblestone plaques covering the face, more prominent over the forehead.

\section{Case Report/Case Presentation}

A 33-week gestation baby boy was born to a healthy consanguineous parent by caesarian section due to premature rupture of the membrane and chorioamnionitis. At birth, he weighed $2.7 \mathrm{~kg}$ (10th centile) and $47.5 \mathrm{~cm}$ in length (10th centile), and his head circumference was $34 \mathrm{~cm}$ (10th centile). His Apgar scores were 7 and 8 at the 1st and 5th min, and he was admitted to the Neonatal Intensive Care Unit due to respiratory distress and was intubated on continuous positive pressure mechanical ventilation. At 32 weeks of gestation, antenatal course of dexamethasone was given to promote lung maturity.

On examination, the baby had no dysmorphic features. His skin examination revealed generalized thick verruciform hyperkeratotic plaques with cobblestone appearance covering all of his body including the scalp with focal areas of hair loss (Fig. 1). Mucous membranes and nails were normal. Ophthalmology examination was normal. His blood eosinophilia at birth was $1,700 \mathrm{cell} / \mathrm{mm}^{3}$, peaked at day 7 reaching $2,300 \mathrm{cell} / \mathrm{mm}^{3}$ and normalized at the age of 1 month. The clinical diagnosis of IPS was rendered and later proven by whole-exome sequencing revealing that he was carrying a homozygous pathogenic variant $(c .1 A>G, p$. Met1 $\mathrm{Val})$ in the $S L C 27 A 4$ gene with parents heterozygous for the same variant.

The proband was managed by using an incubator with $80 \%$ humidity and intravenous hydrocortisone at a dose of $1 \mathrm{mg} / \mathrm{kg} /$ dose $6 \mathrm{~h}$ for 7 days. Skin was managed conservatively by applying frequent, generous amount of petroleum jelly. Over the next 4 weeks, ichthyosis resolved gradually and completely (Fig. 2). The patient developed recurrent pneumonia with recurrent admission to the hospital and was treated with intravenous antibiotics.

\section{Discussion/Conclusion}

IPS is a rare autosomal recessive disorder, first recognized in 1993 [3]. Leaute-Labreze et al. [4] suggested the term "Self-Healing Congenital Verruciform Hyperkeratosis," because skin findings improve spontaneously unlike other forms of congenital ichthyosis, although some patients may persist with skin xerosis or atopy [5]. Neonatal asphyxia is thought to be 
Fig. 2. Photograph of the patient at the age of 1 month showing resolution of ichthyosis.

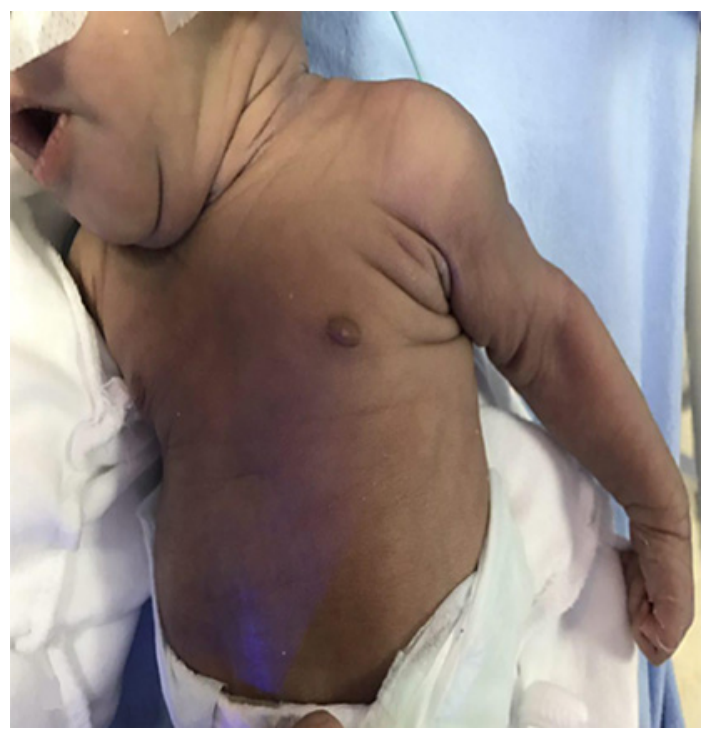

due to aspiration of skin debris that shed into amniotic fluid [5]. Antenatal ultrasound may show separation of chorionic and amniotic membranes and polyhydramnios with starry sky appearance $[4,5]$. Histopathology of skin is pathognomonic with acanthosis, hyperkeratosis, and characteristic aggregates of curved lamellar structures in the perinuclear cytosol of the stratum corneum and stratum granulosum [6]. Perivascular inflammation with eosinophilia was seen in some cases [6].

On electron microscopy, some authors described it as worm-like structures in corneocytes [4]. Transient blood eosinophilia like in our patient has been noticed occasionally [1].

Respiratory complications are the leading cause of death due to inhalation of debris [1]. Considering that lung pathology might be due to aspiring skin debris, we believe that systemic steroids might help mitigating the respiratory complications [7].

IPS was previously reported due to mutation within SLC27A4 that encodes FATP4 [2]. The described $c .1 A>G, p$. Met1Val variant in our patient was previously reported in dbSNP (rs746178942) and in the gnomAD database, resulting in loss of the initiation codon leading to pathological effect on FATP4 protein [8]. The FATP families are transmembrane proteins that transport exogenous fatty acids into cells and activate them. They also function as acyl-CoA synthetases with specificity for very long-chain fatty acids (VLCFA), reducing VLCFACoA synthetase activity and incorporation of VLCFA into neutral and polar lipids [2].

Animal studies suggest that multiple FATPs specifically FATP4 are important for skin barrier function, particularly during embryonic and neonatal period, but are not vital postnatally as other FTAPs may compensate [9]. This may explain spontaneous skin improvement soon after birth [2].

We are presenting and emphasizing the pathognomonic and special cobblestone appearance of ichthyosis in IPS. We also think that systemic steroids may improve the prognosis.

\section{Acknowledgment}

We are grateful to Ms. Zoe Poral Camarig for her secretarial support in proofreading and editing the manuscript. 


\section{Statement of Ethics}

Written informed consent was obtained from the parent/legal guardian of the patient for publication of the details of this medical case and any accompanying images. The case complied with the Declaration of Helsinki. This case is a part of a study approved by the International Review Board (IRB), Ministry of National Guard - Health Affairs (Protocol \# RC20/108/R).

\section{Conflict of Interest Statement}

The authors have no conflicts of interest to declare.

\section{Funding Sources}

There was no funding, and the authors have no conflicts either actual or perceived.

\section{Author Contributions}

Sultan Al-Khenaizan and Asma AlSwailem were involved in contact with the patient, photography, obtaining written consent, and diagnosis of the patient. Mohammed Ali AlBalwi contributed to molecular genetic diagnosis and is the supervisor of the study. All authors contributed to literature review, manuscript writing, and manuscript reviewing.

\section{Data Availability Statement}

All patient data are available in this manuscript.

\section{References}

1 Bygum A, Westermark P, Brandrup F. Ichthyosis prematurity syndrome: a well-defined congenital ichthyosis subtype. J Am Acad Dermatol. 2008;59:S71-4.

2 Klar J, Schweiger M, Zimmerman R, Zechner R, Li H, Törmä H, et al. Mutations in the fatty acid transport protein 4 gene cause the ichthyosis prematurity syndrome. Am J Hum Genet. 2009 Aug;85(2):248-53.

3 Niemi KM, Kuokkanen K, Kanerva L, Ignatius J. Recessive ichthyosis congenita type IV. Am J Dermatopathol. 1993;15:224-8.

4 Léauté-Labrèze C, Boralevi F, Cony M, Maleville J, Lacombe D, Surlève-Bazeille JE, et al. Self-healing congenital verruciform hyperkeratosis. Am J Med Genet A. 2004 Oct 15;130A(3):303-6.

5 Dereksson K, Kjartansson S, Hjartardóttir H, Arngrimsson R. Ichthyosis prematurity syndrome with separation of fetal membranes and neonatal asphyxia. BMJ Case Rep. 2012 Aug 27;2012:bcr0220125823.

6 Khnykin D, Rønnevig J, Johnsson M, Sitek JC, Blaas HG, Hausser I, et al. Ichthyosis prematurity syndrome: clinical evaluation of 17 families with a rare disorder of lipid metabolism. J Am Acad Dermatol. 2012 Apr; 66(4):606-16. Epub 2011 Aug 19.

7 Roberts D, Brown J, Medley N, Dalziel SR. Antenatal corticosteroids for accelerating fetal lung maturation for women at risk of preterm birth. Cochrane Database Syst Rev. 2017;3:CD004454.

8 Inhoff O, Hausser I, Schneider SW, Khnykin D, Jahnsen FL, Sartoris J, et al. Ichthyosis prematurity syndrome caused by a novel fatty acid transport protein 4 gene mutation in a German infant. Arch Dermatol. 2011 Jun; 147(6):750-2.

9 Schmuth M, Ortegon AM, Mao-Qiang M, Elias PM, Feingold KR, Stahl A. Differential expression of fatty acid transport proteins in epidermis and skin appendages. J Invest Dermatol. 2005 Dec;125(6):1174-81.

\section{Karger's}

